\title{
ON THE NONEXISTENCE OF SLICES FOR P-ADIC TRANSFORMATION GROUPS
}

\author{
LAWRENCE M. FRANKLIN
}

ABstract. If a compact Lie group acts effectively on a manifold, then local cross-sections to the orbit structure can be constructed. They are called slices. We prove that if a compact group acts effectively on a manifold, but it is not a Lie group, then a slice cannot exist at every point.

Introduction. Recent papers of R. F. Williams have focused new attention on the Hilbert-Smith conjecture: If a compact group $G$ acts effectively on a manifold, then $G$ is a Lie group. Much interest has centered around properties which arise if one assumes that the conjecture is false, and that the $p$-adic group $A_{p}$ acts effectively on a manifold $M$. The purpose of this paper is to show that if such an action occurs, then for any reasonable definition of a slice, there is at least one point on the manifold at which a slice fails to exist.

Definition. Let $(X, G)$ be a compact $G$-space, and let $x \in X$. A slice at $x$ is a subset $Y$ of $X$ containing $x$ and satisfying:

(i) Whenever $g \in G$ and $y \in Y, g(y) \in Y \Leftrightarrow g \in G_{x}$.

(ii) If $Q$ is an open neighborhood of $e \in G / G_{x}$ which serves as a local cross-section to the cosets of $G_{x}$ at the identity of $G$, then $(g, y) \rightarrow g(y)$ defines a homeomorphism of $Q \times Y$ onto $Q(Y)$. Moreover, $Q(Y)$ is open in $X$.

REMARK. Equivalent definitions of a slice for the case where $G$ is a compact Lie group, may be found in Montgomery and Yang [4], and in Chu [1], and Palais [6], for noncompact Lie groups.

Definition. Let $G_{k}$ denote a cyclic group of order $p^{k}$, and let $g_{k} \in G_{k}$ be a fixed generator. For each pair of integers $i, j \geqq 0$ with $i \geqq j$ we let $\phi_{i j}: G_{i} \rightarrow G_{j}$ be the homomorphism which arises by sending $g_{i} \rightarrow g_{j}$. Note that $\phi_{i i}$ is the identity map on $G_{i}$. With the positive integers as directed indexing set, it is easily verified that the collection of groups and homomorphisms $\left\{G_{i}, \phi_{i j}\right\}$ forms an inverse system. The group $A_{p}=\lim _{\leftarrow}\left\{G_{i}\right\}$ is called the $p$-adic group. Its topology and group operation are those which are induced from the product $X_{i} G_{i}$, and from the standard theorems on inverse limits we find that $A_{p}$ is abelian, Hausdorff, and compact.

Received by the editors March 5, 1970.

AMS 1969 subject classifications. Primary 2240, 5480.

Key words and phrases. Hilbert-Smith conjecture, slice, transformation group, p-adic group. 
We can obtain a large number of facts about $A_{p}$ by constructing it in a different way. In particular we can examine its algebra and topology in more depth. We proceed as follows; consider $A_{p}$ as the set of all sequences

$$
\left(a_{0}, a_{1}, a_{2}, \cdots\right) \text { where } a_{j} \in\{0,1,2, \cdots, p-1\} .
$$

The group operation is performed by adding two sequences coordinate-wise modulo $p$, from left to right, with the carry-over of a one to the next coordinate whenever the sum is greater than or equal to $p$. The identity $e \in A_{p}$ is the sequence $(0,0,0, \cdots)$, and we define a system of neighborhoods $\left\{U_{n}\right\}$ of the identity:

$$
U_{n}=\left\{\left(a_{j}\right): a_{j}=0 \text { for } j<n\right\} .
$$

With this topology we find that $A_{p}$ is totally disconnected, zerodimensional, perfect, and homeomorphic to the Cantor set.

REMARK. The $U_{n}$ are obviously closed subgroups of $A_{p}$, each isomorphic to $A_{p}$. The fact that $p$ is prime is used in an easy proof that the $U_{n}$ are the only closed, nontrivial subgroups of $A_{p}$. The demonstration of the equivalence of the two definitions is a routine exercise in inverse limits.

THEOREM. If the $p$-adic group acts effectively on a manifold $M$, then there is at least one point $X \in M$ at which a slice fails to exist.

We will prove this theorem by a sequence of lemmas, the first of which covers the case where the given action is free. The crucial point centers around finding a point with trivial isotropy group, all points being such if the action is free.

LeммA 1. Let $G=A_{p}$ (the $p$-adic group) act on the locally-connected space $X$. Let $x \in X$ be a point for which the isotropy group $G_{x}$ is trivial. Then there does not exist a slice at $x$.

Proof. We suppose that $Y$ is a slice at $x$. Since $G / G_{x}=G$, we are given an open neighborhood $Q$ of the identity, for which the map $Q \times Y \rightarrow Q(Y)=U$ defined above is a homeomorphism onto an open neighborhood of $x$ in $X$. Since each such open neighborhood $Q$ is a closed subgroup of $G$, isomorphic to $G$, we may assume that $Q=G$. Thus we have a homeomorphism $f: G \times Y \rightarrow U$. We let $V$ be the connected component of $U$ containing $x$, and since $X$ is locally connected, we have that $V$ is open in $X$. The set $f^{-1}(V)$ is open in $G \times X$ and connected. But since $G$ is perfect and totally disconnected, there are no open connected subsets of $G \times X$, which is a contradiction.

Our purpose now is to show that for any effective action of the $p$-adic group on a manifold, there always exists a point which 
satisfies the hypothesis of Lemma 1. An immediate application of Newman's theorem (cf. Montgomery, Samelson and Zippin [3]) gives the result that if a compact abelian Lie group acts effectively on a manifold, then the principal isotropy group is trivial. For a definition of principal isotropy group, and its associated ideas, see Montgomery, Samelson and Yang [2]. From observing that for any action of the $p$-adic on a space $X$, either $G_{x} \subset G_{y}$ or $G_{y} \subset G_{x}$ for any two points $x, y \in X$, we adopt the following:

Definition. If the $p$-adic group $G$ acts effectively on a space $X$, then by a principal point for the action we mean a point $x \in X$ such that $G_{x} \subset G_{y}$ for all $y \in X$.

LEMMA 2. If the p-adic group acts effectively on a manifold $M$, then there is at least one point $x \in M$ which is a principal point for the action. Furthermore, $G_{x}$ is trivial.

Proof. We shall prove that there is a point $x \in M$ for which $G_{x}$ is trivial. We thus suppose that for each point $x \in M, G_{x}$ is nontrivial. We let $g \in G$ be not the identity.

If $H \subset G$ is any nontrivial closed subgroup of $G$, then some power of $g$ belongs to $H$. This is easily seen from our remark following the second definition of the $p$-adic group. Since if we take the closed subgroup $U_{k}$, then $\left(U_{k}\right)^{p}=U_{k+1}$. Thus, for each point $x \in M$, some power of $g$ belongs to $G_{x}$. The map $g: M \rightarrow M$ is then a pointwise periodic homeomorphism, since some power of $g$ belongs to every nontrivial closed subgroup of $G$. By a theorem of Montgomery and Zippin [5], $g$ is periodic. But since $G$ has no elements of finite order, this contradicts the fact that the action was assumed effective.

It is now easily seen that the theorem as stated is an easy consequence of Lemmas 1 and 2, in light of the fact that manifolds are locally connected.

\section{BibLIOGRAPHY}

1. Hsin Chu, On the existence of slices for transformation groups, Proc. Amer. Math. Soc. 18 (1967), 513-517. MR 35 \#3001.

2. D. Montgomery, H. Samelson and C. T. Yang, Groups on $E^{n}$ with $(n-2)$ dimensional orbits, Proc. Amer. Math. Soc. 7 (1956), 719-728. MR 17, 1224.

3. D. Montgomery, H. Samelson and L. Zippen, Singular points of a compact transformation group, Ann. of Math. (2) 63 (1956), 1-9. MR 17, 643.

4. D. Montgomery and C. T. Yang, The existence of a slice, Ann. of Math. (2) 65 (1957), 108-116. MR 19, 291.

5. D. Montgomery and L. Zippin, Topological transformation groups, Interscience, New York, 1955. MR 17, 383.

6. R.S. Palais, On the existence of slices for actions of non-compact Lie groups, Ann. of Math. (2) 73 (1961), 295-323. MR 23 \#A3802.

UNiversity of MARYLAND, BaLtimore, MARYLAND 21228 